\title{
EFECTO DE LOS EXTRACTOS DE Geranium ayavacense $W$. (PASUCHACA) SOBRE LA GLICEMIA EN RATAS CON DIABETES MELLITUS EXPERIMENTAL
}

\author{
José Aranda-Ventura ${ }^{1, a}$, Jorge Villacrés ${ }^{1,2, b}$, Rosario Mego ${ }^{1, c}$, Henry Delgado ${ }^{1, d}$
}

\begin{abstract}
RESUMEN
Objetivos. Determinar si el extracto acuoso liofilizado de Geranium ayavacense (Pasuchaca) tiene algún efecto sobre la glicemia en ratas con diabetes mellitus experimental. Materiales y métodos. La diabetes experimental fue inducida con aloxano. Las ratas cumplieron los criterios: glicemia $>200 \mathrm{mg} / \mathrm{dL}$ posadministración de aloxano y un peso $>200 \mathrm{~g}$. Las ratas con diabetes experimental fueron distribuidas en seis grupos de ocho ratas cada uno. El grupo I recibió $3 \mathrm{~mL}$ de agua destilada (control); el grupo II recibió Geranium ayavacense 12,7 mg/kg; el grupo III recibió Geranium ayavacense 100 mg/kg; el grupo IV recibió Geranium ayavacense 200 mg/kg; el grupo V recibió Geranium ayavacense 300 mg/kg; el grupo VI recibió Geranium ayavacense $500 \mathrm{mg} / \mathrm{kg}$. Se determinó la glicemia basal. Las evaluaciones de la glicemia se realizaron a la 1. a 3 . $^{\mathrm{a}}, 6 .^{\mathrm{a}}, 1$. $^{\mathrm{a}}$ y $24^{\mathrm{a}}$ hora después de administrar las diferentes intervenciones. Resultados. Los grupos de Geranium ayavacense de 300 y $500 \mathrm{mg} / \mathrm{kg}$ disminuyeron significativamente $(p<0,01)$ la glicemia en todas las horas evaluadas después de la administración de los extractos, cuando se compara con el grupo control. El grupo de Geranium ayavacense de $300 \mathrm{mg} / \mathrm{kg}$ decreció su glucosa sanguínea en 8,14; 10,68; 14,87; 19.36 y 23,7\% a la $1 .^{\mathrm{a}}$, , $^{\mathrm{a}}$, $6{ }^{a}, 12 .^{a}$ y $24 .^{a}$ hora respectivamente. Conclusiones. En condiciones experimentales, el extracto acuoso de Geranium ayavacense tiene efecto hipoglicemiante en ratas.
\end{abstract}

Palabras clave: Diabetes mellitus experimental; Plantas medicinales; Glucemia (fuente: DeCS BIREME).

\section{EFFECT OF EXTRACTS OF Geranium ayavacense $W$. (PASUCHACA) ON GLYCEMIA ON RATS WITH EXPERIMENTAL DIABETES MELLITUS}

\begin{abstract}
Objectives. To determine if the lyophilized aqueous extract of Geranium ayavacense (Pasuchaca) has any effect on glycemia in rats with experimental diabetes mellitus. Materials and methods. Experimental diabetes was induced with alloxan. Rats included in the study met the following criteria: glycemia greater than $200 \mathrm{mg} / \mathrm{dL}$ post administration of alloxan, and with a weight greater than $200 \mathrm{~g}$. Rats with experimental diabetes were divided into six groups of eight rats each. Group I received $3 \mathrm{~mL}$ of distilled water (control); group II received Geranium ayavacense $12.7 \mathrm{mg} /$ kg; group III received Geranium ayavacense 100 mg/kg; group IV received Geranium ayavacense $200 \mathrm{mg} / \mathrm{kg}$; group V received Geranium ayavacense $300 \mathrm{mg} / \mathrm{kg}$; group VI received Geranium ayavacense $500 \mathrm{mg} / \mathrm{kg}$. Basal glycemia was determined. Glycemia evaluations were performed at the 1st, 3rd, 6th, 12th and 24th hour after administrating the different interventions. Results. Geranium ayavacense groups of 300 and $500 \mathrm{mg} / \mathrm{kg}$ decreased glycemia significantly $(p<0.01)$ in every hour assessed after administration of the extract, when compared with the control group. Geranium ayavacense group of $300 \mathrm{mg} / \mathrm{kg}$ decreased their blood glucose $8.14 ; 10.68 ; 14.87 ; 19.36$ and $23.7 \%$ in the $1 \mathrm{st}$, 3rd, 6th, 12th and 24th hour respectively. Conclusions. Under experimental conditions, the aqueous extract of Geranium ayavacense has hypoglycemic effects in rats.
\end{abstract}

Key words: Diabetes mellitus, experimental; Plants, medicinal; Blood glucose (source: MeSH NLM).

\footnotetext{
Instituto de Medicina Tradicional, Seguro Social de Salud. Iquitos, Perú.

Facultad de Agronomía, Universidad Nacional de la Amazonia Peruana. Iquitos, Perú.

Médico magíster en salud pública; ${ }^{\mathrm{b}}$ ingeniero agrónomo, magíster en ecología y desarrollo sostenible; ${ }^{\mathrm{C}}$ bióloga; ${ }^{\mathrm{d}}$ químico farmacéutico

Recibido: 04-11-13 Aprobado: 26-02-14
}

Citar como: Aranda-Ventura J, Villacrés J, Mego R, Delgado H. Efecto de los extractos de Geranium ayavacense W. (Pasuchaca) sobre la glicemia en ratas con diabetes mellitus experimental. Rev Peru Med Exp Salud Publica. 2014;31(2):261-6. 


\section{INTRODUCCIÓN}

La diabetes mellitus tipo 2 se caracteriza por hiperglicemia crónica con disturbios en el metabolismo de los carbohidratos, las grasas y las proteínas debido a la resistencia a la insulina y disfunción de las células beta. Los estudios epidemiológicos han confirmado una pandemia global de diabetes tipo 2 , lo cual ha creado una enorme carga para la sociedad, en cuanto a la morbilidad, mortalidad y los gastos de atención de salud (1). Según el Atlas de Diabetes, el 2000 se reportó 151 millones de pacientes ${ }^{(2)}$ y para el 2013 aumento a 382 millones pacientes ${ }^{(3)}$. Esta enfermedad se relaciona con factores de riesgo modificables como la obesidad o el sobrepeso, la inactividad física y los regímenes alimentarios hipercalóricos y de bajo valor nutritivo ${ }^{(4)}$.

Para el tratamiento farmacológico de la diabetes tipo 2, los fármacos clásicos eran las sulfonilureas y la metformina, pero estos fármacos no han logrado detener la progresión de la diabetes tipo 2, de esto se deduce la necesidad de desarrollar nuevos fármacos, es así que progresivamente en los últimos años se han desarrollado las tiazolidinedionas, inhibidores de dipeptidilpeptidasa 4 (iDPP- 4) y los inhibidores del SGLT-2 ${ }^{(1)}$. En la actualidad se buscan y ensayan diversos estrategias médicas mediante una combinación de la dieta, educación, ejercicio físico, inmunoterapia, incluso trasplantes pancreáticos; no obstante la investigación está orientada principalmente en la obtención de nuevas drogas hipoglucemiantes que puedan ayudar al control de la diabetes ${ }^{(5)}$.

A nivel mundial, existen plantas de uso tradicional de las cuales se ha reportado algún efecto hipoglucemiante: la Musa sp. y la Bidens spp. son usadas en el Caribe y el Perú; el Rubus sp. es usado en Nepal; las moras (Morus sp.) se usan en el Mediterráneo y la Mimosa sp. es usada en la India ${ }^{(6)}$. Esta información es importante, por que puede ser el inicio para el desarrollo de nuevos fármacos. También hay información de plantas usadas tradicionalmente para el tratamiento de la diabetes en el norte del Perú, dentro de estas plantas esta el Geranium ayavacense ${ }^{(7)}$. El Geranium ayavacense Willd, es una planta de la sierra que pertenece a la familia Geraniaceae, tradicionalmente se ha reportado que la flor y raíz tienen efecto hipoglicemiante y astringente, así también, es útil en el tratamiento de estomatitis ulcerosa, la gastritis, la gingivitis y lesiones gástricas ${ }^{(8)}$. Con respecto a su composición química, se ha determinado la presencia de taninos, esteroides, flavonoides, antocianinas, antracenos, compuestos reductores, celulosa y almidón ${ }^{(8)}$. Sobre la toxicidad no hay estudios de esta especie, pero sí del extracto acuoso del Geranium sessiliflorum del cual se obtuvo una $C L 50=1472 \mathrm{ug} / \mathrm{mL}$ en Artemia sp., es decir, que a esta concentración el extracto provocó la mortalidad del $50 \%$ de los nauplios de Artemia sp., y según la clasificación de toxicidad por encima de 1000 $\mathrm{ug} / \mathrm{mL}$ se considera no tóxico ${ }^{(9)}$.

Con respecto a estudios farmacológicos de esta especie, no se dispone de reportes en revistas científicas indizadas. Considerando la falta de este tipo de estudios y que la forma de uso tradicional es acuosa, planteamos como objetivo determinar si el extracto acuoso liofilizado de Geranium ayavacense tiene algún efecto sobre la glicemia en ratas con diabetes experimental.

\section{MATERIALES Y MÉTODOS}

\section{PREPARACIÓN DEL EXTRACTO ACUOSO \\ LIOFILIZADO DEL Geranium ayavacense}

La planta denominada pasuchaca procedente de Huaraz en el departamento de Áncash, fue identificada en el Herbarium Amazonense (AMAZ) con el nombre científico de Geranium ayavacense Willd. Ex Kunth, y se registró con el número de herbarium: 41033. Las plantas enteras de esta especie fueron lavadas, limpiadas y cortadas en fragmentos pequeños, luego fueron secadas a una temperatura de $40{ }^{\circ} \mathrm{C}$ por 120 $\mathrm{h}$; a partir del material vegetal seco se procedió a la elaboración del extracto acuoso, el cual se preparó al $10 \%$ utilizando $200 \mathrm{~g}$ del material vegetal seco en 2000 $\mathrm{mL}$ de agua destilada mantenida a una temperatura entre 60 y $70{ }^{\circ} \mathrm{C}$ por $3 \mathrm{~h}$. Posteriormente, se procedió a una filtración y a continuación se congeló a $-20^{\circ} \mathrm{C}$ para luego ser liofilizado mediante una liofilizadora Labotec, modelo $50 \mathrm{~N}$, resultando un polvo fino denominado extracto acuoso liofilizado. El método de elaboración del extracto fue adaptado de un estudio previo ${ }^{(10)}$. Este producto fue almacenado en frascos con tapa hermética a temperatura ambiental y evitando su exposición a la luz solar. Este proceso se desarrolló en el Laboratorio de Farmacognosia del Instituto de Medicina Tradicional.

\section{ESTUDIO FITOQUÍMICO}

La identificación de metabolitos secundarios se realizó a través de pruebas químicas de caracterización (reacción de Dragendorf, reacción de Shinoda, prueba de la espuma, reacción del tricloruro férrico, reacción de la gelatina, reacción de Lieberman-Burchard, reacción de la Ninhidrina y reacción de Molish), según Lock (11).

\section{MODELO EXPERIMENTAL}

Se trabajó con ratas albinas cepa Holtzman, machos sanos, procedentes del Instituto Nacional de Salud 
(Lima-Perú). Las ratas se instalaron en el Bioterio del Instituto de Medicina Tradicional (Iquitos-Perú), en donde fueron aclimatadas durante 15 días a una temperatura entre $26-29{ }^{\circ} \mathrm{C}$, con una humedad entre $45-75 \%$ y un ciclo de luz/oscuridad de 12/12 horas, se les mantuvo con libre acceso al agua y alimentos. Después de la aclimatación, la ratas entre las 13 y 17 semanas de edad alcanzaron un peso > $200 \mathrm{~g}$ (criterio de inclusión para el estudio) y se utilizaron 60 ratas para tomar una muestra de sangre, previo ayuno de $12 \mathrm{~h}$, para determinar las glicemias y verificar si son normales comparándolas con el rango de valores de normalidad según la base de datos de glicemias para ratas albinas cepa Holtzman, machos sanos aclimatadas en el Bioterio del Instituto de Medicina Tradicional (glicemia en ayunas: 70-115 $\mathrm{mg} / \mathrm{dL}$ ). A las ratas cuyas glicemias estuvieron dentro del rango de normalidad, se les indujo la diabetes experimental (hiperglicemia) mediante la administración vía intraperitoneal de aloxano monohidratado (Sigma, Saint Louis, MO, USA) diluido en agua destilada (5\%) a una dosis de $135 \mathrm{mg} / \mathrm{kg}$, este modelo experimental fue adaptado de estudios previos ${ }^{(12,13)}$, luego de esta administración se esperó $48 \mathrm{~h}$, momento en el cual se les tomó una prueba basal de glicemia, previo ayuno de $12 \mathrm{~h}$, para evaluar si el nivel de glicemia se encontraba por encima de $200 \mathrm{mg} / \mathrm{dL}$ (criterio de inclusión que asumimos para considerar diabetes experimental) y todas las ratas que presentaron estos valores fueron incluidas en el estudio para administrarles las sustancias por experimentar. De las ratas evaluadas solo 48 completaron el experimento.

\section{GRUPOS EXPERIMENTALES}

Las 48 ratas que cumplieron los criterios de inclusión fueron distribuidas aleatoriamente en seis grupos de ocho ratas. Todas las sustancias administradas fueron por vía oral mediante cánula intragástrica. Los grupos fueron: Grupo I: agua destilada $3 \mathrm{~mL}$ (control); Grupo II: Geranium ayavacense 12,7 mg/kg; Grupo III: Geranium ayavacense $100 \mathrm{mg} / \mathrm{kg}$; Grupo IV: Geranium ayavacense 200 mg/kg; Grupo V: Geranium ayavacense 300 mg/kg; Grupo VI: Geranium ayavacense 500 mg/kg.

\section{MEDICIÓN DE LA ACTIVIDAD HIPOGLUCEMIANTE}

Teniendo los valores basales hiperglucémicos y habiendo administrado a las ratas las sustancias de experimentación, se efectuaron las evaluaciones de glicemia a la $1 .^{\mathrm{a}}, 3 .^{\mathrm{a}}, 6 .^{\mathrm{a}}, 12$. $^{\mathrm{a}}$ y $24 .^{\mathrm{a}}$ horas posteriores. Durante estas $24 \mathrm{~h}$ de seguimiento, las ratas de los seis grupos no recibieron alimentos, pero sí tuvieron libre acceso al agua. Para la determinación de las glicemias, las muestras de sangre fueron tomadas de la cola de las ratas previa desinfección con alcohol al 70\%, se realizó un corte en la vena caudal en la cola con bisturí 21, se extrajo la sangre con tubos capilares heparinizados con sodio $80 \mathrm{IU} / \mathrm{mL}$, se ocluyó un extremo del capilar con plastilina para ser llevado a la microcentrífuga (Centrifuge Model SH120-1 Kert Laboratory) por 5 min a $12000 \mathrm{rpm}$; terminada la centrifugación se separó el suero de los elementos sanguíneos, se procedió a romper el capilar a nivel del suero, se extrajo $10 \mathrm{uL}$ con una micropipeta (Lab Mate 0,5-10 ul) y se colocó en un tubo de ensayo estéril que contenía $1 \mathrm{~mL}$ del reactivo de glucosa (Glucose Liquicolor Human®), luego se llevó al baño María (NUVE BAHT20) a $37^{\circ} \mathrm{C}$ por $5 \mathrm{~min}$; seguidamente se leyó la absorbancia en el espectrofotómetro (JENWAY 6400) a una longitud de onda de $500 \mathrm{~nm}$. Así se determinó la glicemia según el método GOD-PAP, prueba enzimática colorimétrica para la glucosa, método con desproteinización (Human®).

\section{ANÁLISIS ESTADÍSTICO}

Primero se analizó la normalidad de la variable glicemia a través del test de Shapiro-Wilk, dado que esta variable tuvo una distribución normal se trabajó con promedio y desviación estándar, luego los datos se analizaron con la prueba paramétrica análisis de varianza (ANOVA) de una vía, utilizando el programa estadístico SPSS, versión 20 en español. Se consideró significativo $p<0,05$. Al realizar la prueba de homogeneidad de varianza usando el estadístico de Levene $(p<0,05)$, se evidenció que las varianzas no son homogéneas. Como el ANOVA nos mostró una diferencia significativa, a continuación se aplicó la prueba de comparaciones múltiples de Dunnet porque la intención era comparar un grupo control con el resto de tratamientos y porque las varianzas no son homogéneas. Se consideró significativos a $p<0,05$ y altamente significativo a $p<0,01$. Se usó Microsoft excel 2010 para calcular las diferencias porcentuales.

\section{ASPECTOS ÉTICOS}

Se respetaron las recomendaciones para el manejo de animales de experimentación según lo estipulado en la Directiva Europea 2010/63/EU para la protección de animales de laboratorio. Previo a realizar la eutanasia, las ratas fueron sedadas con ketamina $5 \mathrm{mg} / \mathrm{kg}$ vía intramuscular y luego fueron sacrificadas por el método de dislocación cervical ${ }^{(14)}$.

\section{RESULTADOS}

En el estudio fitoquímico preliminar se determinó predominantemente alcaloides, compuestos fenólicos, flavonoides, taninos y saponinas caracterizados mediante las pruebas de Dragendorf, de tricloruro 
Tabla 1. Estudio fitoquímico preliminar del extracto acuoso liofilizado de Geranium ayavacense $W$.

\begin{tabular}{llc}
\hline Prueba de caracterización & $\begin{array}{c}\text { Metabolito } \\
\text { secundario }\end{array}$ & Resultado \\
\hline Reacción de Dragendorf & Alcaloides & ++ \\
Reacción Shinoda & Flavonoides & ++ \\
Prueba de la espuma & Saponinas & ++ \\
Reacción del tricloruro ferrico & $\begin{array}{l}\text { Compuestos } \\
\text { fenólicos }\end{array}$ & ++ \\
Reacción de la gelatina & Taninos & ++ \\
Reacción de la Lieberman- & Esteroides o & - \\
Burchard & terpenoides & - \\
Reacción de la ninhidrina & $\begin{array}{l}\text { Aminoácidos } \\
\text { libres }\end{array}$ & - \\
Reacción de la Molish & Glicósidos & - \\
\hline
\end{tabular}

férrico, de Shinoda, de la gelatina y de la espuma respectivamente, en el extracto acuoso liofilizado del Geranium ayavacense (Tabla 1).

Las ratas seleccionadas tuvieron un peso de 262,06 $\pm 21,04$ g y sus valores de glicemia antes de la inducción de la diabetes experimental fueron de 90,67 $\pm 11,91 \mathrm{mg} / \mathrm{dL}$, estos valores están dentro del rango de normalidad según la base de datos de valores de glicemia para estos animales de experimentación del Bioterio del Instituto de Medicina Tradicional -EsSalud. Con la inducción del aloxano se logró valores hiperglicémicos de 259,12 \pm 21,24 mg/ dL. Ver las medias y sus desviaciones estándar de las glicemias por grupo y horas en Tabla 2. El análisis de varianza ANOVA reveló diferencia significativa $(p<0,01)$ entre los tratamientos. El grupo V (Geranium ayavacense $300 \mathrm{mg} / \mathrm{kg}$ ) y VI (Geranium ayavacense 500 $\mathrm{mg} / \mathrm{kg})$ disminuyeron significativamente $(p<0,01$, prueba de Dunnet) la glicemia en todas las horas evaluadas posadministración del extracto, cuando se compara con el grupo I (control). El grupo de IV (Geranium ayavacense $200 \mathrm{mg} / \mathrm{kg}$ ) disminuyó significativamente $\left(p<0,01\right.$, prueba de Dunnet) la glicemia solo a la $6 .^{a}$ hora posadministración del extracto al compararlo con el grupo I (control). El grupo II (Geranium ayavacense $12,7 \mathrm{mg} / \mathrm{kg}$ ) y III (Geranium ayavacense $100 \mathrm{mg} / \mathrm{kg}$ ) no tuvieron efecto significativo ( $p>0,05$, prueba de Dunnet) sobre los niveles de glicemia, al compararlo con el grupo I (control) (Tabla 2).

El grupo V (Geranium ayavacense 300 mg/kg) disminuyó la glicemia en 8,$1 ; 10,7 ; 14,9 ; 19,4$ y $23,7 \%$ a la $1 .{ }^{a}, 3 .^{a}$, 6. ${ }^{a}, 12 .{ }^{a}$ y $24 .{ }^{a}$ hora, respectivamente; mientras que el grupo VI (Geranium ayavacense 500 mg/kg) disminuyó la glicemia en 7,$1 ; 9,6 ; 9,5 ; 20,2$ y $26,5 \%$ a la $1 .^{a}, 3 .^{a}$, 6. ${ }^{a}, 12 .^{a}$ y $24 .^{a}$ hora, respectivamente. Ver la diferencia porcentual de las glicemias de cada grupo, con respecto al basal en Tabla 3. También se evidencia que los grupos V y VI (Geranium ayavacense de 300 y 500 mg/kg) disminuyen progresiva y sostenidamente la glicemia desde la $1 .^{\text {a }}$ hora hasta las $24 \mathrm{~h}$ posadministración de los extractos.

\section{DISCUSIÓN}

En el presente estudio se puso en evidencia que el extracto acuoso liofilizado de Geranium ayavacense a la dosis de 300 y 500 mg/kg (grupo V y VI respectivamente) tiene algún efecto hipoglucemiante en ratas macho albinas cepa Holtzman con diabetes mellitus experimental, cuando se compara con el grupo I (control). Ambos grupos empiezan su acción a partir de la $1 .^{a}$ hora, y la respuesta hipoglucemiante es sostenida hasta las 24 h posadministración del extracto; conforme pasa el tiempo es mayor la disminución de la glicemia. No se evidenció un efecto dosis-respuesta en los grupos $\mathrm{V}$ y VI, excepto en la evaluación de las 24 h. Ambos grupos lograron una disminución significativa de la glicemia durante todas las horas de evaluación con respecto al grupo I (control), sin embargo, no fue suficiente para normalizar los niveles de glicemia. Para el caso del grupo IV (Geranium ayavacense de

Tabla 2. Promedios de glicemia (mg/dl) basal y post administración de agua destilada y Geranium ayavacense en ratas con diabetes mellitus experimental

\begin{tabular}{|c|c|c|c|c|c|c|c|}
\hline \multirow{2}{*}{$\begin{array}{c}\text { Grupo de } \\
\text { tratamiento }\end{array}$} & \multirow{2}{*}{ Dosis } & \multicolumn{6}{|c|}{ Tiempo } \\
\hline & & Basal & $1 \mathrm{H}$ & $3 \mathrm{H}$ & $6 \mathrm{H}$ & $12 \mathrm{H}$ & $24 \mathrm{H}$ \\
\hline Grupo I (AD) & $3 \mathrm{ml}$ & $242,51 \pm 36,86$ & $248,96 \pm 24,34$ & $243,67 \pm 15,75$ & $254,62 \pm 23,44$ & $252,56 \pm 20,44$ & $239,01 \pm 18,96$ \\
\hline Grupo II (GA) & $12,7 \mathrm{mg} / \mathrm{Kg}$ & $272,76 \pm 8,41$ & $283,96 \pm 7,71$ & $288,06 \pm 9,17$ & $278,97 \pm 11,63$ & $275,52 \pm 9,81$ & $264,44 \pm 16,28$ \\
\hline Grupo III (GA) & $100 \mathrm{mg} / \mathrm{Kg}$ & $253,96 \pm 17,17$ & $262,31 \pm 5,20$ & $257,77 \pm 8,28$ & $249,5 \pm 12,13$ & $261,72 \pm 6,57$ & $240,76 \pm 10,04$ \\
\hline Grupo IV (GA) & $200 \mathrm{mg} / \mathrm{Kg}$ & $264,44 \pm 9,02$ & $260,06 \pm 10,16$ & $255,01 \pm 12,45$ & $239,87 \pm 29,16^{*}$ & $256,6 \pm 16,13$ & $223,76 \pm 32,36$ \\
\hline Grupo V (GA) & $300 \mathrm{mg} / \mathrm{Kg}$ & $253,9 \pm 12,69$ & $233,23 \pm 17,05^{*}$ & $226,79 \pm 16,77^{*}$ & $216,15 \pm 16,25^{*}$ & $204,74 \pm 38,75^{*}$ & $193,72 \pm 54,08^{*}$ \\
\hline Grupo VI (GA) & $500 \mathrm{mg} / \mathrm{Kg}$ & $268,4 \pm 15,99$ & $249,46 \pm 18,79^{*}$ & $242,66 \pm 23,18^{*}$ & $243,01 \pm 41,36^{*}$ & $214,21 \pm 60,67^{*}$ & $197,28 \pm 70,90^{*}$ \\
\hline
\end{tabular}

Los valores representan la media \pm desviación estándar, $n=8$ en cada grupo, AD: agua destilada, GA: Geranium ayavacense, H: Hora

Análisis estadístico ANOVA de una vía seguido de la prueba de Dunnet para comparaciones multiples

${ }^{*} p<0,01$, estadísticamente significativo frente al grupo control. 
Tabla 3. Diferencia porcentual (\%) de la glicemia a diferentes dosis de Geranium ayavacense y agua destilada con respecto a la glicemia basal en ratas con diabetes mellitus experimental

\begin{tabular}{lccccccc}
\hline \multirow{2}{*}{ Grupo de tratamiento } & \multirow{2}{*}{ Dosis } & \multicolumn{7}{c}{ Tiempo } \\
\cline { 3 - 8 } & & Basal & $\mathbf{1 ~ H}$ & $\mathbf{3 ~ H}$ & $\mathbf{6 ~ H}$ & $\mathbf{1 2} \mathbf{H}$ & $\mathbf{2 4} \mathbf{~ H}$ \\
\hline Grupo I (AD) & $3 \mathrm{ml}$ & 100 & 2,7 & 0,5 & 4,9 & 4,1 & $-1,4$ \\
Grupo II (GA) & $12,7 \mathrm{mg} / \mathrm{Kg}$ & 100 & 4,1 & 5,6 & 2,3 & 1,0 & $-3,1$ \\
Grupo III (GA) & $100 \mathrm{mg} / \mathrm{Kg}$ & 100 & 3,3 & 1,5 & $-1,8$ & 3,1 & $-5,2$ \\
Grupo IV (GA) & $200 \mathrm{mg} / \mathrm{Kg}$ & 100 & $-1,7$ & $-3,6$ & $-9,3$ & $-2,9$ & $-15,4$ \\
Grupo V (GA) & $300 \mathrm{mg} / \mathrm{Kg}$ & 100 & $-8,1$ & $-10,7$ & $-14,9$ & $-19,4$ & $-23,7$ \\
Grupo VI (GA) & $500 \mathrm{mg} / \mathrm{Kg}$ & 100 & $-7,1$ & $-9,6$ & $-9,5$ & $-20,2$ & $-26,5$ \\
\hline
\end{tabular}

$\mathrm{AD}$ : agua destilada, GA: Geranium ayavacense, $\mathrm{H}$ : hora

$200 \mathrm{mg} / \mathrm{kg}$ ), si bien se evidencia una reducción de la glicemia en todas las horas de evaluación en relación con el grupo I (control), no hubo diferencia significativa excepto a la $6 .{ }^{\text {a }}$ hora.

La presencia de flavonoides en el extracto acuoso liofilizado del Geranium ayavacense podría ser la causa de su efecto hipoglucemiante, ya que existen estudios sobre un tipo de glicósido de flavona (derivados de flavonoide) que demostró actividad hipoglucemiante debido a que estaría ligado a los receptores proliferadores de peroxisomas (PPAR) o antagonistas de receptores de glucagón, inhibidor dipeptidil peptidasa IV y activador de los receptores de insulina ${ }^{(15)}$. Hay tres subtipos de PPAR: PPAR- $\alpha$, PPAR-y y PPAR- $\delta$; con respecto al PPAR-y está relacionado con la regulación y maduración del adipocito, siendo el sitio de acción para los fármacos sensibilizadores a la acción de la insulina, como la pioglitazona, troglitazona y rosiglitazona, los que conllevan a la reducción de la glicemia $(16,17)$. Esta molécula podría ser el punto de acción donde probablemente actuarían los flavonoides presentes en el extracto acuoso liofilizado de Geranium ayavacense. Además, se ha demostrado que los flavonoides, como la quercetina, ejercen buen efecto hipoglucemiante, a través de la acción sobre la alfaglucosinasa que favorece la fosforilación de la glucosa, para formar glucosa-6 fosfato, paso limitante para la glucólisis y la regulación de la glicemia ${ }^{(18)}$. Los alcaloides son otro grupo de metabolitos encontrados en el extracto acuoso del Geranium ayavacense, los cuales estarían coadyuvando con el efecto hipoglucémico; al respecto, se ha reportado un estudio con el Geranium lechleri, en donde concluyen que los alcaloides constituyen los principios activos responsables del efecto hipoglucemiante ${ }^{(13)}$; por otro lado, hay reportes que los alcaloides son los responsables del efecto hipoglucemiante de algunas plantas, así, por ejemplo, los alcaloides quinolizidine del Lupinus perennis realzan la liberación de insulina inducida por la glucosa, desde los islotes celulares aislados de ratas ${ }^{(19)}$.
Otro factor que podría haber contribuido a la reducción de la glicemia, sería que el extracto acuoso de Geranium ayavacense actúe como un inhibidor de la alfaglucosidasa, se hace este planteamiento basado en que se ha demostrado que el extracto metanólico del Geranium dielsiaum inhibe la elevación de la glicemia por inhibición de la actividad de la alfa-glucosidasa, en un modelo de mamífero que refleja el estado in vivo ${ }^{(20)}$. En el contexto de la información publicada y estos hallazgos, planteamos que el efecto hipoglucemiante del Geranium ayavacense podría ser explicado por la acción sinérgica de sus alcaloides, flavonoides y otros metabolitos secundarios presentes en el extracto acuoso liofilizado de la planta total.

Algunas limitaciones deben ser reconocidas. Para evaluar el efecto hipoglucemiante se usó un modelo agudo, el cual solo permite evidenciar este efecto dentro de las $24 \mathrm{~h}$ siguientes a la inducción de la diabetes, pero es un modelo válido por que permite hacer un screening rápido de nuevas sustancias con potencial acción inmediata sobre el control de la glicemia; además, hay estudios que evalúan la glicemia durante $24 \mathrm{~h}{ }^{\left({ }^{(1)}\right.} \mathrm{e}$, incluso, en menos tiempo ${ }^{(18,21)}$. Para controlar la modificación de la glicemia causada por el estrés, se recomienda incluir un grupo control negativo, es decir, un grupo de ratas a las cuales no se les induzca diabetes y se les suministre agua destilada, lo cual no se hizo. Consideramos que un buen grupo control debe estar expuesto a las mismas condiciones que el resto de grupos para poder comparar, es así que incluimos como control a un grupo de ratas a las cuales se les indujo diabetes y se les administró agua destilada; cabe señalar que hay estudios que incluyen este tipo de grupo control ${ }^{(13,18)}$.

En conclusión, el extracto acuoso liofilizado de la Geranium ayavacense a la dosis de 300 y $500 \mathrm{mg} / \mathrm{kg}$ tiene algún efecto hipoglucemiante en ratas con diabetes mellitus experimental. Con ambas dosis se evidenció un efecto hipoglucemiante sostenido a partir de la $1 .{ }^{a}$ hora prolongándose hasta la $24 .{ }^{a}$ hora posadministración del extracto. 
Agradecimientos: al licenciado en Estadística Eliseo Zapata Vásquez de la Universidad Nacional de la Amazonía Peruana, por su asesoramiento en el análisis estadístico. A los estudiantes Romel Tuesta, Charles Domínguez y Teddy Torrejón de la Facultad de Farmacia y Bioquímica de la Universidad Nacional de la Amazonía Peruana, por su colaboración en la manipulación de los animales de experimentación.

Contribuciones de autoría: JAV participó en la concepción y diseño del estudio, en el análisis e interpretación de datos, redacción del manuscrito, revisión crítica del artículo y la aprobación de la versión final a publicar. JV contribuyó con la información botánica y recolección de las especies vegetales materia del presente estudio, apoyo logístico y revisión crítica del artículo. RM contribuyó con los análisis de glicemia, manipulación de los animales de experimentación y con la recolección de resultados. HD contribuyó con el estudio fitoquímico de la especie vegetal, manipulación de los animales de experimentación y con la recolección de resultados.

Fuentes de financiamiento: Instituto de Medicina Tradicional del Seguro Social de Salud EsSalud, Iquitos, Perú.

Conflictos de interés: los autores declaran no tener conflictos de interés.

\section{REFERENCIAS BIBLIOGRÁFICAS}

1. Tsang MW. The management of type 2 diabetic patients with hypoglycaemic agents. ISRN Endocrinol. 2012;2012:478120. doi: $10.5402 / 2012 / 478120$.

2. Colagiuri R. Diabetes: a pandemic, a development issue or both? Expert Rev Cardiovasc Ther. 2010 Mar;8(3):305-9. doi: 10.1586/erc.10.12.

3. International Diabetes Federation. IDF: Diabetes Atlas [Internet]. Bruselas: IDF; 2013 [citado el 27 enero de 2014]. Disponible en: http://www.idf.org/ diabetesatlas

4. Organización Panamericana de la Salud. Métodos para la prevención y el control de la obesidad y de la diabetes. Región de las Américas. Epidemiological Bulletin [Internet]. 2008 [citado el 28 de mayo de 2013];27(2):[aprox 6 p.] Disponible en: http://new.paho.org/bulletins/index. php?option $=$ com_content $\&$ task $=$ view \&id=195\& $\&$ Itemid $=272$

5. Esquivel-Gutiérrez E, Noriega-Cisneros R, Bello-González M, SaavedraMolina A, Salgado-Garciglia R. Plantas utilizadas en la medicina tradicional mexicana con propiedades antidiabéticas y antihipertensivas. Biológicas. 2012;14(1):45-52.

6. Bussmann RW. The globalization of traditional medicine in Northern Perú: from shamanism to molecules. Evid Based Complement Alternat Med. 2013;2013:291903.

7. Bussmann RW, Glenn A. Traditional knowledge for modern ailments - plants used for the treatment of diabetes and cancer in Northern Perú. J Med Plants Res. 2011;5(31):6916-30.

8. Mendocilla M, Villar M. Monografía de plantas medicinales. En: Villar M, Villavicencio O, ed. Manual de fitoterapia.
Lima: Seguro Social de Salud-EsSalud: 2001. p. 265-267.

9. Bussmann RW, Malca G, Glenn A Sharon D, Nilsen B, Parris B, et al. Toxicity of medicinal plants used in traditional medicine in Northern Peru. J Ethnopharmacol.2011Sep 1;137(1):121 40. doi: 10.1016/j.jep.2011.04.071.

10. Pérez M, Jiménez E, Boffill M, González D, Méndez R, Verdecía B, et al. Toxicidad aguda de un extracto acuoso de Boldoa purpurascens Cav. en el modelo de sube y baja en ratas. Rev Cubana Plant Med. 2008;13(2).

11. Lock O. Investigación fitoquímica, métodos en el estudio de productos naturales. 2da ed. Lima: Pontificia Universidad Católica del Perú; 1994.

12. Nimenibo-Uadia R. Effect of aqueous extract of Canavalia ensiformis seeds on hyperlipidaemia and hyperketonaemia in alloxan-induced diabetic rats. Biokemistri. 2003;15(1):7-15.

13. Castañeda $B$, Castro de la Mata $R$, Manrique R, Ibañez L, Fujita R, Barnett L, et al. Estudio fitoquímico y farmacológico de 4 plantas con efecto hipoglicemiante. Horiz Med. 2008; 8(1):6-34.

14. The European Parliament and The Council of The European Union. Directive 2010/63/EU of The European Parliament and of The Council of 22 September 2010 on the protection of animals used for scientific purposes: Annex IV Methods of killing animals. Official Journal of the European Union [Internet]. Strasbourg, Francia, September 22, 2010 [citado el 14 de Febrero de 2014]. Disponible en: http://eur-lex.europa.eu/LexUriServ/ LexUriServ.do?uri=OJ:L:2010:276:0033 :0079:En:PDF

15. Arroyo J, Martínez J, Ronceros G, Palomino R, Villareal A, Bonilla P, et al.
Efecto hipoglicemiante coadyuvante del extracto etanólico de hojas de Annona muricata L (guanábana), en pacientes con diabetes tipo 2 bajo tratamiento con glibenclamida. An Fac med. 2009;70(3):163-7.

16. Asgary S, Naderi G, Sarrafzadegan N, Ghassemi N, Boshtam M, Rafie M, et al. Anti-oxidant effect of flavonoids on hemoglobin glycosylation. Pharm Acta Helv. 1999 Feb;73(5):223-6.

17. Feinstein DL, Spagnolo A, Akar C, Weinberg G, Murphy P, Gavrilyuk V, et al. Receptor independent actions PPAR thiazolidinedione agonists: Is mitochondrial function the key? Biochem Pharmacol. 2005 Jul;70(2):177-88.

18. Castañeda B, Castro R, Puebla P, Ibañez L. Efecto del extracto atomizado de las hojas de Calophyllum brasiliense "lagarto caspi” sobre la glicemia. Horiz Med. 2011;11(1):7-14.

19. Jung M, Park M, Lee HC, Kang YH, Kang ES, Kim SK. Antidiabetic agents from medicinal plants. Curr Med Chem. 2006;13(10):1203-18.

20. Karato M, Yamaguchi K, Takei S, Kino T, Yazawa K. Inhibitory effects of pasuchaca (Geranium dielsiaum) extract on alphaglucosidase in mouse. Biosci Biotechnol Biochem. 2006 Jun;70(6):1482-4.

21. Kulkarni YA, Veeranjaneyulu A. Effects of Gmelina arborea extract on experimentally induced diabetes. Asian Pac J Trop Med. 2013 Aug;6(8):602-8. doi: 10.1016/S1995-7645(13)60104-2.

Correspondencia: José Aranda Ventura

Dirección: Av. Abelardo Quiñones 2265,

Iquitos, Perú

Teléfono: (51)965766025

Correo electrónico:jose.aranda@essalud.gob.pe 